\title{
A New Model to Predict Weak Lensing Peak Counts
}

\section{Chieh-An Lin and Martin Kilbinger}

\author{
Service d'Astrophysique, CEA Saclay, \\ Orme des Merisiers, Bât 709, 91191 Gif-sur-Yvette, France \\ email: chieh-an.lin@cea.fr
}

\begin{abstract}
Peak statistics from weak gravitational lensing have been shown to be a promising tool for cosmology. Here we propose a new approach to predict weak lensing peak counts. For an arbitrary cosmology, we draw dark matter halos from the halo mass function, and calculate the number of peaks from the projected halo mass distribution. This procedure is much faster than time-consuming $N$-body simulations. By comparing these "fast simulations" to $N$-body runs, we find that the peak abundance is in very good agreement. Furthermore, our model is able to discriminate cosmologies with different sets of parameters, using high signal-to-noise peaks $(\gtrsim 4)$. This encourages us to examine the optimal combinations of parameters to this approach in the future.
\end{abstract}

Keywords. peak counting, mass function, weak lensing

\section{Introduction}

Weak gravitational lensing (WL) uses the deflection of light from background galaxies to probe the Universe. It contains the information about the non-linear regime which has been encoded in cosmological structures. Peak statistics from WL is a measure of this non-Gaussian information. Peaks are local maxima of the projected mass distribution, therefore they probe the mass function, which is sensitive to cosmology.

To predict WL peak counts, Fan et al. (2010, hereafter FSL10) gave an analytical model using Gaussian random field theory. Their model computes the peak number density function by taking into account Gaussian shape noise. Meanwhile, Maturi et al. (2010) provided another analytical model by defining peaks as a contiguous area, and considering aperture-mass peaks with different filter functions. However, analytical models rely on linear filters to suppress noise, which may not be optimal. Furthermore, such models are strongly limited in more realistic scenarios, e.g. in the presence of galaxy intrinsic alignment. This motivated us to adopt a new approach, which is probabilistic.

The idea is to create "fast simulations" using a halo sampling technique. This avoids time-consuming $N$-body simulations and keeps open the possibility for using non-linear filters. The only requirement is a cosmology with a known mass function.

\section{The model}

The fast simulations are generated by sampling halos from the mass function of Jenkins et al. (2001), in 10 redshift bins from 0 to 1 . These halos are randomly placed across the field of view, thus their angular positions are not correlated. The NFW density profile is chosen.

We validate this approach by comparing our fast simulations to $N$-body runs and two intermediate cases as follows: full $N$-body runs (case 1 ); replacing $N$-body halos with 
NFW profiles with the same masses (case 2); randomizing angular positions of halos from the case 2 (case 3 ); fast simulations, corresponding to our model (case 4 ). In the case 2 and the case 3 , the halo population and their redshift are identical to $N$-body runs. This allow us to study impacts from two hypothesis on which our model is based: (1) diffuse, unbound matter, for example filaments, does not significatly contribute to peak counts, and (2) the spatial correlation of halos has a minor influence on peak counts.

In this study, we follow Hamana et al. (2004) and add the shape noise as Gaussian noise with variance $\sigma_{\text {pix }}^{2}=\sigma_{\epsilon}^{2} /\left(2 n_{\mathrm{g}} A_{\text {pix }}\right)$, where $\sigma_{\epsilon}=0.4$ is the intrinsic ellipticity dispersion of our choice, $n_{\mathrm{g}}$ is the galaxy number density, and $A_{\mathrm{pix}}$ is the surface of a pixel. To supress the noise, we applied a Gaussian filter function $W_{\mathrm{G}}(\boldsymbol{\theta})=\exp \left(-\theta^{2} / \theta_{\mathrm{G}}^{2}\right) / \pi \theta_{\mathrm{G}}^{2}$, with $\theta_{\mathrm{G}}=1$ arcmin.

We measure peaks as local maxima on a gridded convergence $(\kappa)$ map. A peak is defined to have a signal-to-noise ratio (SNR) higher than its 8 neighbors. The SNR is given by $\nu=\kappa / \sigma_{\text {noise }}$ where $\sigma_{\text {noise }}^{2}=\sigma_{\epsilon}^{2} /\left(4 \pi n_{\mathrm{g}} \theta_{\mathrm{G}}^{2}\right)$. Finally, we compress the information from the WL maps into abundance histograms of peak counts.

\section{Simulations}

The $N$-body simulations "Aardvark" used in this study are provided by A. Evrard and generated by GADGET-2 (Springel 2005). The Aardvark parameters had been chosen to be a WMAP-like $\Lambda$ CDM cosmology, with $\Omega_{\mathrm{m}}=0.23, \Omega_{\Lambda}=0.77, \Omega_{\mathrm{b}}=0.047, \sigma_{8}=0.83$, $h=0.73, n_{\mathrm{s}}=1.0$, and $w_{0}=-1.0$.

Halos were identified using ROCKSTAR (Behroozi et al. 2013). The field of view is $859 \mathrm{deg}^{2}$. Ray-tracing for the case 1 was performed with CALCLENS (Becker 2013), available only on a subset of $53.7 \mathrm{deg}^{2}$ (16 times smaller). For the other cases, raytracing is performed on the entire field. Source galaxies are regularly placed at redshift $z_{\mathrm{s}}=1.0$, and their number density $n_{\mathrm{g}}$ is $21.69 \mathrm{arcmin}^{-2}$.

\section{Results and conclusion}

Fig. 1 shows the SNR histograms from the 4 studied cases, from which we can make the following observations. First, all cases agree with each other in low $\nu$ bins, since peaks with $\nu \lesssim 3 \sim 4$ are dominated by noise (see FSL10). Second, the randomization of halo angular positions is a good approximation (compare the green circles to the red squares). Third, our model is in a good agreement with $N$-body simulations, except for very high SNR.

The left panel of Fig. 2 gives a comparison between FSL10 and this study. Overall, our model performs better than FSL10. The right panel is a sensitivity test with different inputs of $\Omega_{\mathrm{m}}$ and $\sigma_{8}$. One can observe that different cosmologies are discernible for $\nu \gtrsim 4$ and that our model is accurate enough to distinguish between cases.

As a result, we provide a new model which can accurately recover lensing peak abundance. Our model opens the possibility to model non-linearly reconstructed convergence fields. It allows us to study optimal combinations of parameters sensitive to this approach. In this proceeding, the analysis is done for a flat universe without galaxy intrinsic alignment. However, further studies for more complex scenarios are in the progress. 


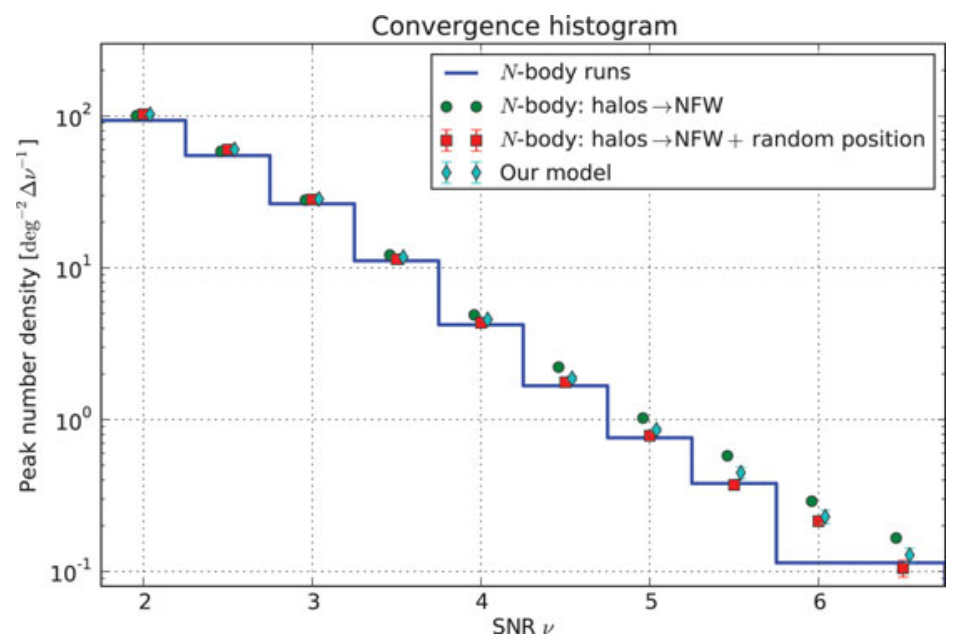

Figure 1. SNR histogram for 4 studied cases. Blue solid line: $N$-body runs; green circles: replacement of halos by NFW profiles; red squares: replacement of halos by NFW profiles and randomization of halo angular positions; cyan diamonds: our model. The error bars are standard deviations calculated from 8 different realizations. The $N$-body runs corresponds to $53.7 \mathrm{deg}^{2}$, while for the other cases the field of view is $859 \mathrm{deg}^{2}$.
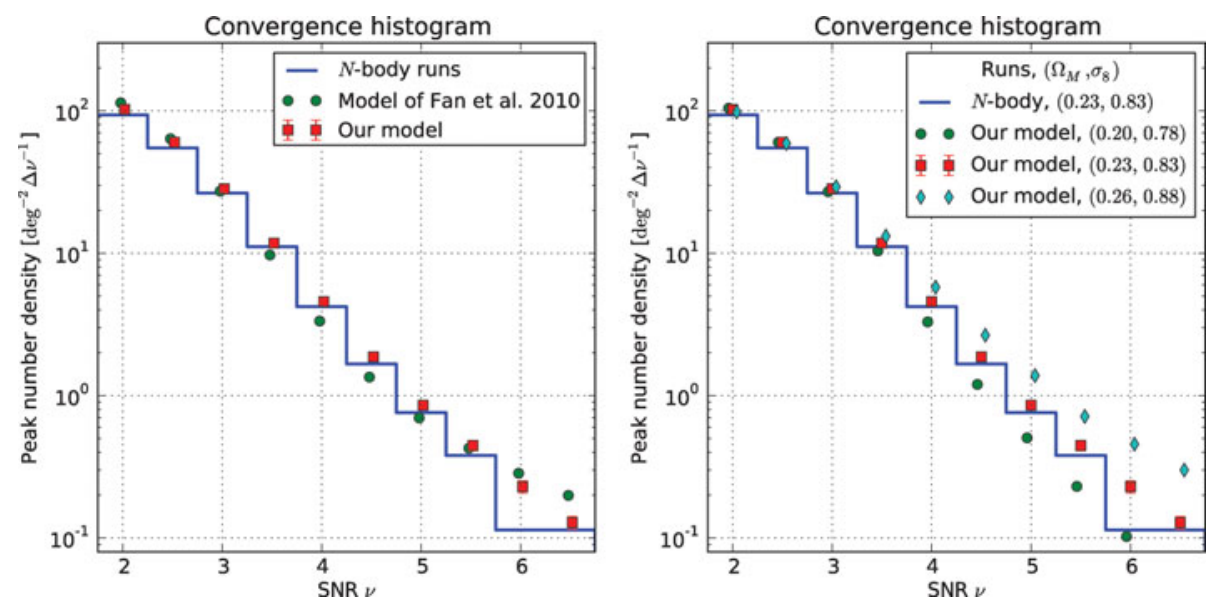

Figure 2. Left: comparison between the model from Fan et al. 2010 and this study. Right: the sensitivity test on $\left(\Omega_{\mathrm{m}}, \sigma_{8}\right)$. In both plots, the blue solid line corresponds to the $N$-body runs, and the red squares are our model with the same parameters as the $N$-body simulations.

\section{References}

Becker, Matthew R. 2013, MNRAS, 435, 115

Behroozi, P. S., Wechsler, R. H., \& Wu, H.-Y. 2013, ApJ, 762, 109

Fan, Z., Shan, H., \& Liu, J. 2010, ApJ, 719, 1408

Hamana, T., Takada, M., \& Yoshida, N. 2004, MNRAS, 350, 893

Jenkins, A., Frenk, C. S., White, S. D. M., Colberg, J. M., Cole, S., Evrard, A. E., Couchman,

H. M. P., \& Yoshida, N. 2001, MNRAS, 321, 372

Maturi, M., Angrick, C., Pace, F., \& Bartelmann, M. 2010, A\& A, 519, A23

Navarro, J. F., Frenk, C. S., \& White, S. D. M. 1996, ApJ, 462, 563

Springel, V. 2005, MNRAS, 364, 1105 